\title{
Comparing the performance between Virtual Machines and Containers using deep learning credit models
}

\author{
Kennedy Chengeta \\ kennedy.chengeta@kaributechs.com \\ University of Cape Town \\ Cape Town, South Africa
}

\begin{abstract}
The cloud computing paradigm utilizes neutralization to isolate data, workloads and network bandwidth in an elastic mode. The providers hence have historically used Virtual Machines (VM) to perform this workload isolation. However these virtual machines require huge resources and are costly to set-up and deploy. Containerization has of late been used as a different approach for neutralization albeit as a Software as a service approach. Docker containerization has been one of the most popular approaches and its a solution where containers all share the same resources and operating system as opposed to virtual machines which use hyper-visor technology to abstract hardware and operating systems. The study uses different cloud vendors to compare docker containers for response time, download time, CPU processing time as well as memory usage against virtual machines. The study did a performance comparison for Virtual Machines and Docker Containers in various cloud providers namely AWS, Google Cloud as well as Microsoft Azure cloud platforms. The dataset used included deep learning big data downloaded from Kaggle website and classified for loan defaulting with Keras and tensorflow python implementation frameworks. The comparisons do prove that docker containers are faster than KVMs and Xen virtual machines. The study also proves that by using Kubernetes framework for scaling the containers, the performance of the docker containers improves compared to the docker and bare metal as well as cloud frameworks.
\end{abstract}

CCS Concepts: • Containerization $\rightarrow$ Docker Containers; • Clustering $\rightarrow$ Kubernetes; • Hosting $\rightarrow$ Virtual Machines; $\bullet$ Cloud $\rightarrow$ Microsoft Azure.

\footnotetext{
Permission to make digital or hard copies of all or part of this work for personal or classroom use is granted without fee provided that copies are not made or distributed for profit or commercial advantage and that copies bear this notice and the full citation on the first page. Copyrights for components of this work owned by others than ACM must be honored. Abstracting with credit is permitted. To copy otherwise, or republish, to post on servers or to redistribute to lists, requires prior specific permission and/or a fee. Request permissions from permissions@acm.org.

icARTi '21, December 9-10, 2021, Virtual Event, Mauritius

() 2021 Association for Computing Machinery.

ACM ISBN 978-1-4503-8575-6/21/12 ..\$15.00

https://doi.org/10.1145/3487923.3487934
}

Keywords: containers, deep learning, kubernetes, performance, open stack, VM (Virtual Machines)

\section{ACM Reference Format:}

Kennedy Chengeta. 2021. Comparing the performance between Virtual Machines and Containers using deep learning credit models. In International Conference on Artificial Intelligence and its Applications (icARTi '21), December 9-10, 2021, Virtual Event, Mauritius. ACM, New York, NY, USA, 8 pages. https://doi.org/10.1145/3487923. 3487934

\section{Introduction}

Virtual machines provide computing resources and allow for hardware abstraction and consolidation of resources. They are also popular for hosting Virtual Defined Functions or VNFs, reduce vendor lock-in and improved security. In cloud computing, services like Amazon Web Services, Google Cloud and Microsoft Azure the importance of virtualization in provision of infrastructure as a service, abstraction and resource allocation has been witnessed due to rapid growth $[2,11]$. The virtualization technologies however, add extra layers of virtualization at the costs of performance and costs for customers. Containers have been introduced as alternatives to virtual machines due to their low utilization of computational resources. These technologies bring near native performance in cloud and on premise environments. Key container services include docker containers, Amazon EC2 Containers, Google Container Engine and container orchestrations like Kubernetes. Virtual machines include Amazon EC2 virtual machines, OpenStack virtual machines, VMware ESXi, Vsphere and Vcenter [2, 3, 10, 17]. Primary motivations for containers have been largely to encapsulate and easier deploying of systems, run micro-service architecture components, efficiency as well as flexibility.

The study aims to study the performance of traditional virtual machines and the container technologies. Critical areas of assessment include performance metrics, CPU utilization, booting time, throughput and scaling, evaluation of overhead the two technologies on bare metal directly. The study will also look at performance by combining the two technologies where containers are executed in virtual machines to see if they give better throughput both for on premise and cloud 
environments.

The study will compare the output of executing big data based on deep learning resource intensive classification on both containers and virtual machines. The output will be collected and results compared for disk, CPU, memory, network and application performance using the resource intensive machine learning application running deep learning classification of big data loan lending dataset. The study details the objectives, literature study, methodology, implementation, results analysis and conclusion.

\section{Literature Review}

Virtualization which built the foundations of cloud computing enabled resource provisioning, multi-tenancy, system constitution in IAAS or infrastructure as a service and was mostly based on hypervisor virtual platforms to be a middle-ware between the CPU and operating system. Different studies analysed the use of virtual machines in abstracting hardware and exposing as infrastructure as a service components. With emergency of cloud computing, hypervisor based virtual machines like OpenStack, KVM (Kernel Virtual Machine), VirtualBox and Vmware.Virtual machines are known to degrade over time through slow performance because of disk fragmentation, virtual machine sprawling, memory and CPU bottlenecks as well non frequent patch updates [3, 10, 11, 22]. Key considerations for performance in virtual machines include memory, CPU and storage disk sizes. as well as network access to external systems as well as high traffic peak periods scalability. In virtualization, a hypervisor runs on the underlying hardware and guests will use the underlying hardware features.

Containers are a major enabler in operating system virtualization which are alternate to virtual machines and have been found to be demanding low computational resources and are mostly classified as application containers, docker or system containers [3]. They eliminate need of an extra guest operating system.

Various studies have looked at container based and virtual machines comparisons with focus on performance, cloud computing, WebRTC servers and resource allocation [23]. The study notes however lack of comparisons of systems of big data machine learning applications on virtual machines and containers focusing on resource usage, scaling, set-up costs, memory and CPU utilizations. Big data applications include the use of frameworks like deep learning, Spark and Hadoop for big data classification using large datasets.

Hypervisors are grouped into Type 1 and Type 2 hypervisors with the former directly linked to the infrastructure hardware and the later runs as an application on the host application namely HyperKit or OSX and Hyper-V and Virtual Box and VMWare. For docker containers the docker daemon replaces the hypervisors and have docker images as binaries and the images are managed independently by the docker daemon. The daemon rationalises resources and isolates host OS from containers and it requires less memory and storage.

\section{Virtual Machines}

Popular virtual machines include KVM, VMware ESXi, Hyper$\mathrm{V}$ and XEN and are both hypervisor based virtual machines as opposed to LXC and Docker which are container based platforms [1, 11, 23]. The following section discusses Xen and KVM virtual machines. The two were selected based on their widespread use and popularity in the virtual machine community as well as their widespread documentation availability.

\subsection{Xen}

The Xen hypervisor supports x86, itanium and x86-64 as well as ARM architectures and runs on linux as well as windows and is primarily used by Citrix and Oracle Virtual Machines. With Xen, full virtualization is enabled and it runs directly on the hardware and allocates memory and CPU as well as hardware resources $[1,12]$. The running instance will have drivers of all system software it needs. The guest domains run their own software and handle all the access for input and output as Type 1 hypervisors [11]. The Virtual Machines provide abstraction and operational efficiency and reduce costs to data centers and Xen also uses para-virtualization where the operating system is aware of the virtualization and works with the hypervisor to improve operational efficiency. The Xen and KVM machines are compared in the following figure 1.
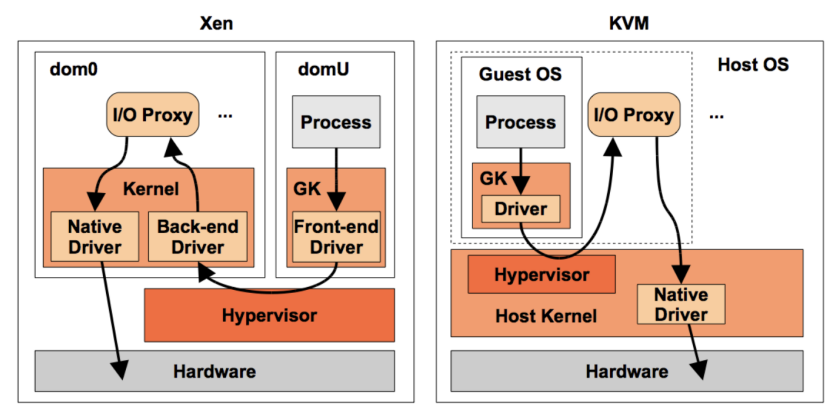

Figure 1. Comparison of the Xen and KVM virtual machines [21]

\subsection{Kernel Virtual Machines}

KVM (for Kernel-based Virtual Machine) enable complete virtualization solutions on $\mathrm{x} 86$ architecture with Intel VT or AMD-V hardware and is open source based. The kernel is 
based on Linux and acts as a Type 2 Hypervisor and enables improved performance by coordinating calls for processor memory and hard disks as well as network resources through the host operating system [1, 12]. The KVM can be loaded and run on multiple virtual machines on solitary servers running unchanged Linux as well as Windows. Multiple virtual machines can be run unchanged on Linux or Windows images with every VM or virtual machine having dedicated hardware, network and graphics drivers. The KVM architecture is shown in figure 2 showing the user and guest modes.

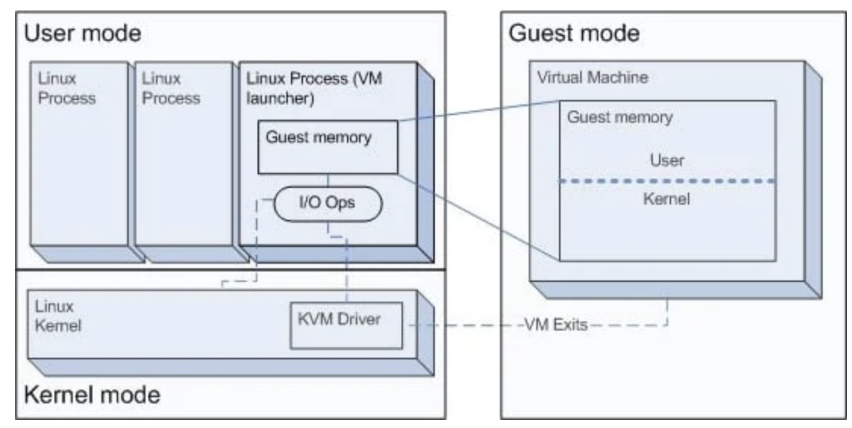

Figure 2. Architecture of the Kernel Virtual Machines [6]

Both Xen and KVM are both open source modifications of the Linux kernel and KVM runs as a kernel module. Xen supports both paravirtualization as well as hypervisor.

\section{Containerization}

As alternative to virtualization is containerization with technologies like LXC or Linux containers and docker being prominent [20, 21]. They are less memory intensive and enable of micro-services and require less storage. To enable self automation, deployment and scaling containers have also been scaled using technologies like Kubernetes or K8s. Popular container technologies are explained in the following section.

\subsection{LXC (Linux Containers)}

LXC (Linux Containers) enables operation system virtualization to execute several solitary Linux system containers positioned on controller hosts supported on a singular Linux kernel [25]. The kernel enables cgroups to allow limitation and priority of CPU, memory and network resources without starting up virtual machines. It enables namespace isolation and has mounted file systems as well as networking and process trees. Early versions of the Docker containers were based on LXC.

\subsection{Docker}

Docker improves on LXC with kernel as well as APIs to enable processes execute in isolation with CPU processing unit, memory,input and output, network and other resources
$[13,19,26]$. The Docker namespaces are used to isolate application views of base operating environment as well as file systems, user ids and process trees. The containers are created with images and an action happens when the image is being built. For docker images, each action command is above the prior action and execution of commands is maunal or systematically based on docker files with scripts of list of commands and arguments. Docker images can also be executed in virtual machines as well.

\subsection{Differences between Containerization and Virtualization}

Some of the differences between containerization and virtualization include the fact containers run on host operating system and are normally combined with the kernel of the host OS and virtual machines run on hypervisor [10, 22, 23]. For virtual machines like KVM there is full virtualization from hardware to the OS and docker is implemented on the cloud without guest OS and economic resources. and is ideal for micro-hosting services. Both technologies are used in cloud computing in IaaS cloud models and easier bundling of applications is achieved with docker containers and images $[20,21]$.

Virtualization virtual machines are more preferred if its needed to create a secure system and for high availability and scalable system containers are used. Docker containers focus on applications and dependencies whereas virtual machines introduce flexibility. and VMs provide better portability, security and isolation and docker provide better SaaS for application and software portability to given users. The hypervisor and containers are compared in the following figure 3.

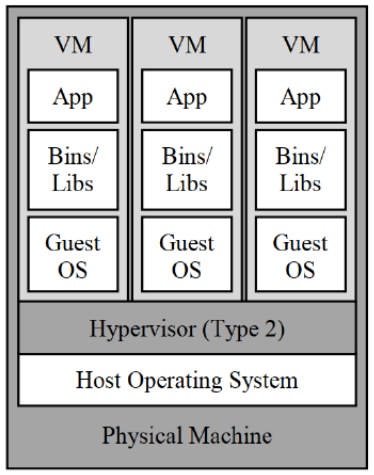

(a) Hypervisor-based virtual service.

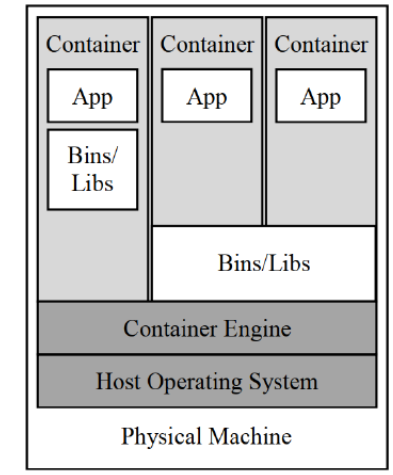

(b) Container-based virtual service.
Figure 3. Hypervisor compared to the container based virtual services [13]

Hybrid options where both virtual machines and docker containers are used can give both benefits. Other difference 
include the following summary. The performance and variances of the two approaches are summarized in the following table 1 .

Table 1. Comparisons of virtual machines and container systems

$\begin{array}{lll}\text { Variance } & \text { Virtual Machines } & \text { Containers } \\ \text { Operating System } & \text { Need a guest } & \text { Shared } \\ \text { Booting Speed } & \text { Slower than traditional machine } & \text { Faster than VMs } \\ \text { Standardization } & \text { OS standards specific in nature } & \text { Application specific in nature } \\ \text { Portability } & \text { Not very portable } & \text { Faster and easily ported } \\ \text { Servers needed } & \text { Needs more } & \text { Few servers } \\ \text { Security } & \text { Hypervisor defines security } & \text { Security is shared } \\ \text { Redundancy Level } & \text { VM owns resources } & \text { Shared OS so less redundancy } \\ \text { Hardware Abstraction } & \text { Hardware Abstracted } & \text { Hardware access can be achieved } \\ \text { Resources Sharing } & \text { Huge resources needed } & \text { Less resources needed and shared } \\ \text { Memory } & \text { Huge memory footprint } & \text { Less memory footprint and shared } \\ \text { Files sharing } & \text { File sharing not possible } & \text { File sharing possible }\end{array}$

\section{Performance of Docker Containers and Virtual Machines}

Various studies have been done to compare docker and virtual machines including the use of Cassandra dataset by Shrinibab. Key conclusions from the study include that containers had lower overheads than VMWare virtualized version and the performance was as good as non virtualized versions. In $[6,17]$, the authors measured various central processing unit CPU power, disk storage intensive docker processes and the latter the found correlation of performance of cgroup file and the /proc file systems when workloads were increased on the CPU.

Raho et al [26] compared KVM to Xen hypervisors using ARM architecture based docker containers andmade conclusions that much improvments on performances on the loads resulted and networking for containers whilst hypervisors shows better disk I/O and TCP streaming performances.Zhang et al [26] compared performance of hypervisor based virtualization and container based in high performance computing. and the latter gave better performance results. Walters et al [25] used VMWare ESXi , KVM as well as Xen to open source LXC containers and achieved 98 percent accuracy on base performance and virtual machines ranging only 2 percentage points only. Kozhirbayev and Sinnott [13] compared the container based technologies in the cloud environment and the Ferreira [8] study compared Docker, Flockport (LXC) and VMs. Few studies have used machine learning to compare docker containers and virtual machines as big data analysis to see their performance and accuracy. Few studies have also compared bare metal against cloud environments to see the performance of the the two virtualization approaches

\subsection{Kurbenetes and OpenShift}

Kubernetes is a popular open source deployment and scaling engine for management of container based deployments which in this cases are docker containers [5, 8, 9, 16]. The design includes a pod as a unit of application. The pod houses at least one docker container which are grouped together using identical IP as well as physical or virtual storage. The cluster also has a master node and group of the worker nodes $[7,9,16]$.

The master node also called the control plane manages the issues in the cluser and also has the stcd, kube-apiserver, kube controller as well as kube scheduling component. The etcd is a storage repository for all configuration and cluster state data as shown in in the next figure with two worker nodes 4 pods and exposed using the API server in the master node. The frontend is the kubernetes API server to expose the kubernetes control plane. It is through which a client can communicate with the kubernetes system as shown in figure 4 .

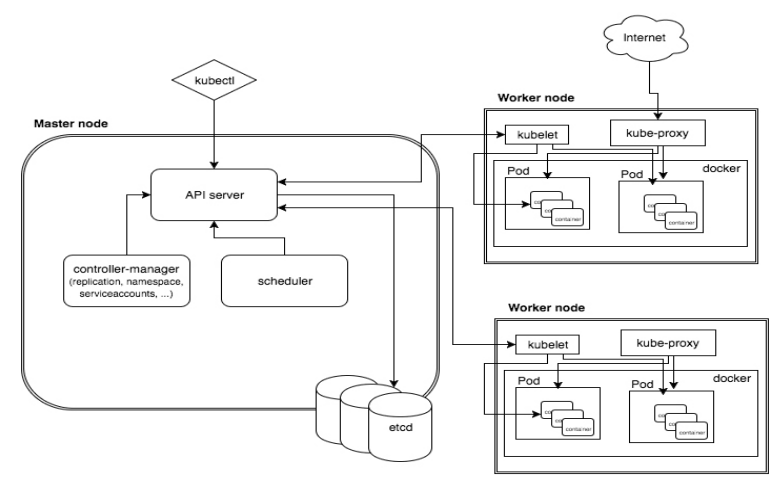

Figure 4. Diagram showing Kubernetes architecture components [9]

The unscheduled pods are assigned by the scheduler based on resource avaialability, session affinity configurations and the controller manages the cluster state machine for instance the replica quantities. Comparisons have been done in edge servers [7, 24], on managed services with containers [9] and for resource management in kubernetes [16]. The interaction with a cloud enviroment is shown in figure 5.

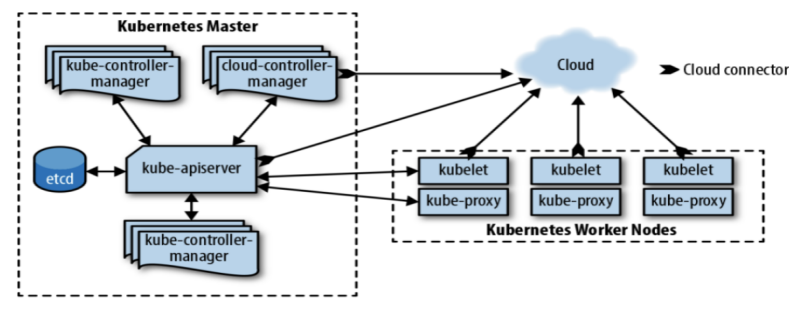

Figure 5. Kubernetes cluser connected to a cloud provider like Azure or Amazon Web Services [5] 
The docker swarm and open shift are other options [15]. The docker swarm hosts the containers virtually and uses the docker daemon for communication to scale to more hosts.

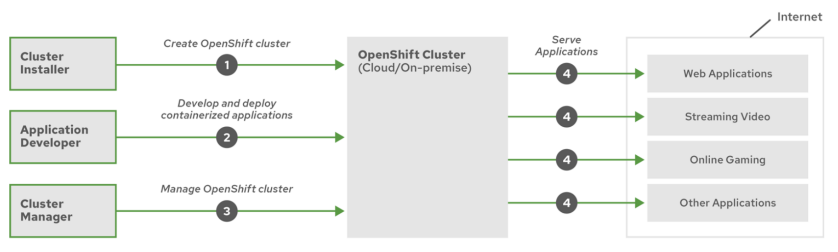

Figure 6. Redhat openshift $[15,18]$

5.1.1 Red Hat OpenShift infrastructure architecture. Redhat open shift uses infrastructure nodes based on kubernetes architecture $[14,18]$ as shown in figure 6 . The nodes will house the cluster services, log aggregation as well as metric and reporting information in some form of registry as well as network routing data [15].

\section{Motivation of the Study}

Various studies have compared virtual machines and containers for performance but the study offers a different approach of analysing deep learning machine big data algorithms on virtual machines and docker containers on Google Cloud Platform, Microsoft Azure and Amazon Web Services using EC2 instances as well as bare metal on premise hardware. The deep learning implementation uses the keras and tensorflow python implementation using credit data set. The approach was to classify the credit lending dataset for loan defaulting with two class attributes namely defaulting or not defaulting. The study also enhances the container technology by using a Kubernetes cluster to boost its performances based on the docker containers. The virtual machines used include KVM and Xen based hypervisors all with Ubuntu linux.

\section{Methodology}

The study uses deep learning credit scoring data to predict defaulting of Kaggle sourced data of home credit lending loans based on Convolution Neural Networks and CNN-RNN or recurrent neural networks. The classification experiments using deep learning are executed on the virtualized AWS, Azure, Google Cloud and OpenStack environments both on and off the cloud as well using different docker containers like Amazon EC2 Container Service and Google Cloud Container. A java front-end to execute the deep learning classification is developed using Java and react web technology frameworks. Open source tools and cloud monitoring tools like Cloudwatch and cloud trail are used to monitor and measure the performance disk utilization, network latency, degradation, CPU usage and boot up times as well when there is an application which is resource intensive like deep learning is executing.

The classification results are stored in an elastic search environment and a dashboard comparing the results using Kibana is created and compared. Because OpenStack supports both bare metal and virtual machines as well as container based The study will compare results from the three stack layers. OpenStack supports bare metal provisioning, virtual machines and container based servers. Load testing and stress testing will also be applied using the home loans lending dataset on both virtual and container technologies. The Convolutional Neural Networks algorithms were based on the Deep learning convolutional neural networks (CNN) implemented using keras and tensorflow. The date went through various training and testing and a series of convolution of layers with filters and kernels. With $\mathrm{CNN}$, more than one convolution layers with different pooling and fully connected layers are used and is represented by the following equation

$$
S(i, j)=(K * I)(i, j)=\sum_{m} \sum_{n} I(i-m, j-n) K(m, n)
$$

Convolutional networks are a breed of neural network based on convolution in the middle layers. and also represented in the following form for the input kernel.

$$
\text { input } \left.\left.\otimes \text { kernel }=\sum_{v=0}^{\text {columns }}\left(\sum_{w=0}^{\text {rows }} \operatorname{input}(w-a, v-b) \operatorname{kernel}(w, v)\right)\right)\right)
$$

Thee deep learning approach used followed a Keras and TensorFlow architecture as follows where Keras is the high level language in python.

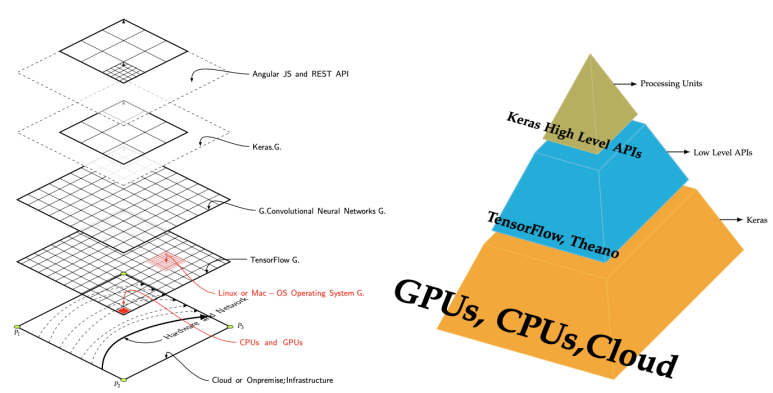

Figure 7. Deep Learning with Keras and TensorFlow architecture

\section{Approach}

The comparison approach of the Virtual Machine and container based on the cloud solutions like Amazon Web Services, Microsoft Azure and Google Cloud and bare metal approach. For monitoring tools like AppDynamics based agent, Jmeter and Elastic Search Kibana were used to monitor the 
performance of the study. The study used deep learning classification approach by analysing credit data to predict loan defaulting for the performance assessment of the containers and virtual machine approaches and then based time and response times.

Data: Preprocess Prosper and lending club deep data downloaded from Kaggle

Result: Default/no default loan class attributes while For each virtual machine/container do

1. Classify the training and test sets for Prosper and Lending Club;

2. For each dataset in AWS, Microsoft Azure and Google cloud as well as bare metal

3. Apply download the dataset and pre-process and classify;

6. Measure the CPU usage, network bandwidth as well download speed for Docker and VMs

End While'

end

Algorithm 1: Preprocess credit lending data for VM and Container comparisons

\subsection{Deep Learning Dataset used for performance comparisons}

For the deep learning classification, the peer to peer credit lending dataset was used and included a mix of Prosper Lending and Lending Club datasets. The number of of observations included around 40000 observations for Lending club and 100000 for Prosper Loan dataset. The implementation was done in python and downloaded from the Kaggle dataset with the downloading of the Kaggle dataset also measured for performance with the docker and virtual machines configured. Epochs of 500-1000 were used and the learning rate was base lined at 0.0001 . A sample of the Lending Club dataset is shown below where the loan status was shown against different statuses and total payment done as shown in figure 8 For the Prosper Lending dataset the results are shown as follows and the data shows the loan borrowers and their prosper ratings as in figure 8 .

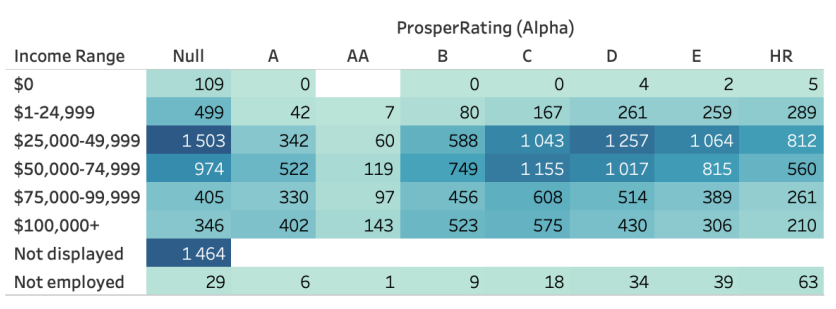

Figure 8. Prosper rates versus income range

\section{Results Analysis}

Different results were measured including the following measured on an Azure Cloud infrastructure based on running the peer to peer lending dataset on different containers as well as virtual machines showing the following response times. The confusion matrix for various classifiers are shown in the following two graphs in the following figure.

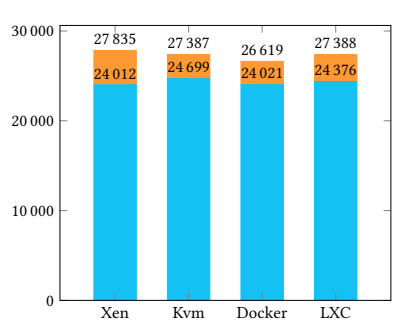

No Defaulting Defaulting

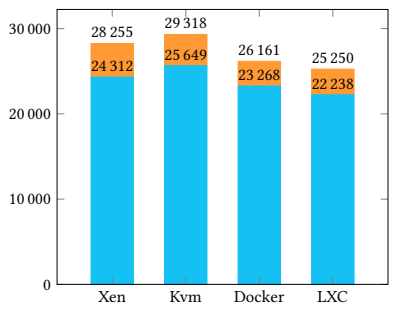

No Defaulting Defaulting
Figure 9. Prosper and Lending Club on the first and subsequent graph

The previous graph in figure 9 show that bulk of results had no defaulting and both graphs used deep learning approaches with same datasets hence the results were almost similar. The dataset used including use of 90 percent for training at random and 10 percent classification which explains for the slight differences. The deep learning classification results given include the following showing the accuracy rate of 0.988 and 0.99 percent on the two credit peer to peer datasets.
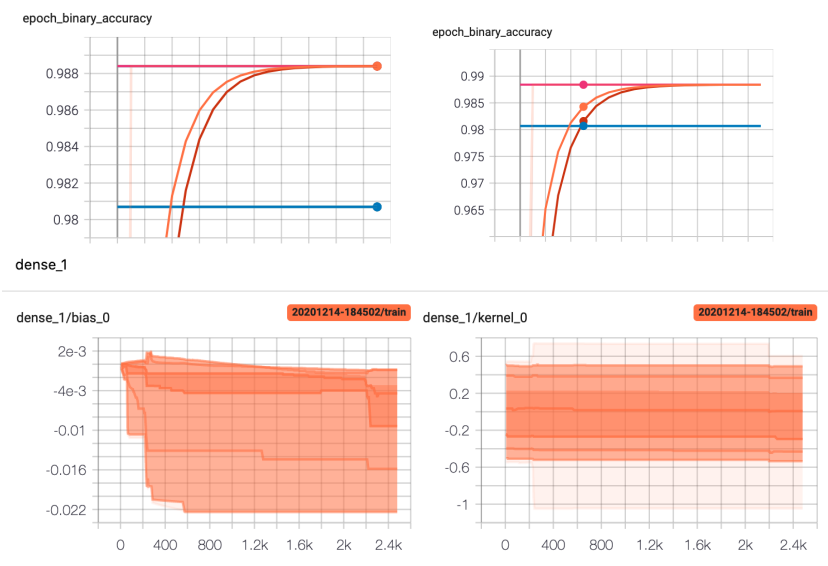

Figure 10. Keras and tensorflow

The virtual machines used in the study include KVM and Xen and Docker containers were used for the comparisons with also configured in a kubernetes cluster. Microsoft Azure Cloud environment classification using the VMs and container results are shown below. 


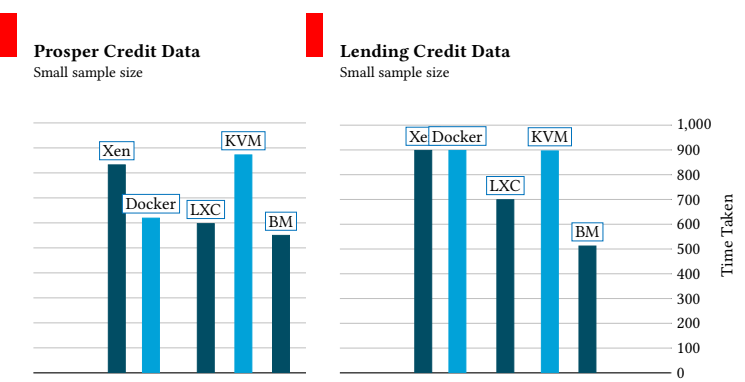

Figure 11. Various datasets using Lending as well as Prosper Credit Dataset.

AWS Cloud environment classification using the VMs and container results are shown below

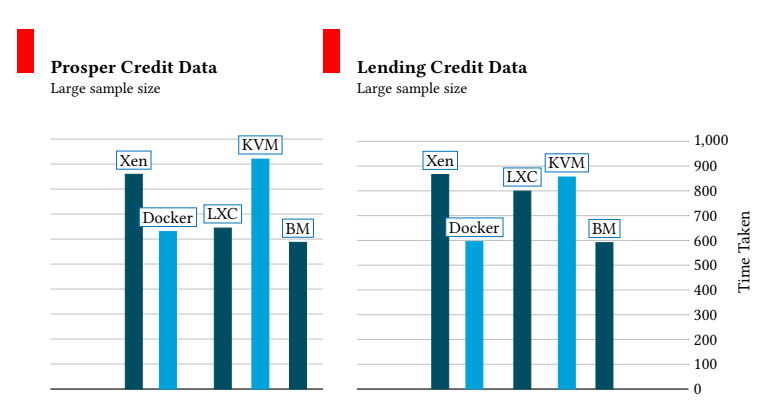

Figure 12. Various datasets using Lending as well as Prosper Credit Dataset.

Google Cloud Platform classification using the VMs and container results are shown below

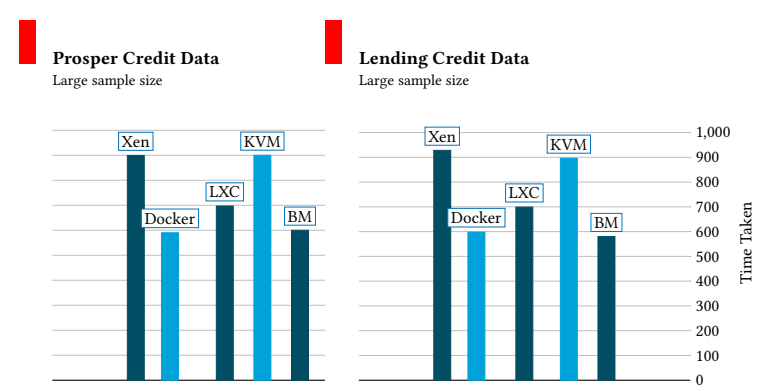

Figure 13. Various datasets using Lending as well as Prosper Credit Dataset.

\subsection{Comparing CPU, Disk Utilization and other factors for Virtual Machines and Docker Containers}

The following tables compared the booting time, network delay as well as data download and network delay for the 4 different virtualization approaches based on the deep learning dataset of Prosper Lending and Lending Club datasets for classifications.
Table 2. $\operatorname{lr}=0.001$ and Lending Club Dataset

\begin{tabular}{lllll} 
Function & KVM & Xen & Docker & Docker(Kuber) \\
\hline Booting Time sec & $\mathrm{x}$ & +0.040 & -0.400 & -0.350 \\
Network Delay ms & $\mathrm{x}$ & +0.623 & -0.412 & -0.611 \\
New VM Creation Time ms & $\mathrm{x}$ & +2.321 & -0.882 & -1.343 \\
CPU Ave Usage \% & $\mathrm{x}$ & +0.070 & -0.060 & -0.110 \\
Dataset Download time(s) & $\mathrm{x}$ & +3.230 & -5.110 & -6.310 \\
Disk Utilization(\%) & $\mathbf{x}$ & $\mathbf{+ 0 . 0 5 0}$ & $\mathbf{- 0 . 0 8 0}$ & $\mathbf{- 0 . 0 9 0}$ \\
Memory Utilization(\%) & $\mathbf{x}$ & $\mathbf{+ 0 . 0 6 0}$ & $\mathbf{- 0 . 0 8 0}$ & $\mathbf{- 0 . 1 2 0}$
\end{tabular}

For Prosper dataset, the following results show different comparison metrics the study used to do the comparisons showing booting time, network time and dataset download relative time where $x$ is the baseline value of the KVM relative to the others.

Table 3. $1 r=0.0001$ and Prosper Dataset

\begin{tabular}{lllll} 
Function & KVM & Xen & Docker & Docker(Kuber) \\
\hline Booting Time sec & $\mathrm{x}$ & +0.020 & -0.500 & -0.650 \\
Network Delay ms & $\mathrm{x}$ & +0.345 & -0.234 & -0.465 \\
New VM Creation Time ms & $\mathrm{x}$ & +2.654 & -0.994 & -1.245 \\
CPU Ave Usage \% & $\mathrm{x}$ & +0.060 & -0.050 & -0.090 \\
Dataset Download time(s) & $\mathrm{x}$ & +2.450 & -4.560 & -5.670 \\
Disk Utilization(\%) & $\mathbf{x}$ & $\mathbf{+ 0 . 0 4 0}$ & $\mathbf{- 0 . 0 3 0}$ & $\mathbf{- 0 . 0 5 0}$
\end{tabular}

The results show better performance of the two datasets in the AWS and Google Cloud compared to the Azure cloud environments. The docker container technologies fared better than the two virtualized technologies KVM and Xen in all the three cloud environments used in the study. The booting time for the AWS EC2, Google cloud virtual machines and Azure environments were averaged and the Xen took the longest to start-up with worst network delay of 0.345 relative to the KVM and almost half a second less than the docker and docker on kubernetes cluster. The docker container showed better download times than KVM and Xen for both the Lending Club and Prosper credit datasets.

\section{Conclusion}

Docker container was compared virtual machine (VM) and the results show that docker images take less memory and are faster compared to the virtual machines used in the study namely KVM and Xen. Different cloud platforms namely Google Cloud Platform, Amazon Web Services AWS platform as well as the Microsoft Azure platform were used in the performance comparisons. The best performance was witnessed in the Amazon AWS cloud environment. The study also concludes that enhancing the docker containers with Kubernetes increases the performance by few percentage points and reduces the delay as well as quickens deployment time as well and reduced Quality of Service transaction speed. The study could have used GPU units or quantum computing hardware but this was discovered as prohibitive in terms of access and cost. 


\section{References}

[1] Luca Abeni and Dario Faggioli. 2019. An experimental analysis of the xen and kvm latencies. In 2019 IEEE 22nd International Symposium on Real-Time Distributed Computing (ISORC). IEEE, 18-26.

[2] Rabindra K Barik, Rakesh K Lenka, K Rahul Rao, and Devam Ghose. 2016. Performance analysis of virtual machines and containers in cloud computing. In 2016 international conference on computing, communication and automation (iccca). 1204-1210. https://doi.org/10.1109/ CCAA.2016.7813925

[3] Rabindra K. Barik, Rakesh K. Lenka, K. Rahul Rao, and Devam Ghose. 2016. Performance analysis of virtual machines and containers in cloud computing. In 2016 International Conference on Computing, Communication and Automation (ICCCA). 1204-1210. https://doi.org/10. 1109/CCAA.2016.7813925

[4] Rabindra K Barik, Rakesh K Lenka, K Rahul Rao, and Devam Ghose. 2016. Performance analysis of virtual machines and containers in cloud computing. In 2016 international conference on computing, communication and automation (iccca). IEEE, 1204-1210.

[5] Brendan Burns, Joe Beda, and Kelsey Hightower. 2019. Kubernetes: up and running: dive into the future of infrastructure. O'Reilly Media.

[6] Emiliano Casalicchio and Vanessa Perciballi. 2017. Auto-scaling of containers: The impact of relative and absolute metrics. In 2017 IEEE 2nd International Workshops on Foundations and Applications of Self* Systems $\left(\right.$ FAS $\left.^{*} W\right)$. IEEE, 207-214.

[7] Halim Fathoni, Chao-Tung Yang, Chih-Hung Chang, and Chin-Yin Huang. 2019. Performance comparison of lightweight kubernetes in edge devices. In International Symposium on Pervasive Systems, Algorithms and Networks. Springer, 304-309.

[8] Wes Felter, Alexandre Ferreira, Ram Rajamony, and Juan Rubio. 2015 An updated performance comparison of virtual machines and linux containers. In 2015 IEEE international symposium on performance analysis of systems and software (ISPASS). IEEE, 171-172.

[9] Arnaldo Pereira Ferreira and Richard Sinnott. 2019. A performance evaluation of containers running on managed kubernetes services. In 2019 IEEE International Conference on Cloud Computing Technology and Science (CloudCom). IEEE, 199-208.

[10] Khasa Gillani and Jong-Hyouk Lee. 2020. Comparison of linux virtual machines and containers for a service migration in $5 \mathrm{~g}$ multi-access edge computing. ICT Express 6, 1 (2020), 1-2.

[11] Mohd Badrulhisham Ismail, Habibah Hashim, and Yusnani Mohd Yusoff. 2017. Dynamics resource allocation via virtual machine for cloud computing: Statistical approach. In 2017 7th IEEE International Conference on System Engineering and Technopreneurship (ICE2T). 1-5. https://doi.org/10.1109/ICE2T.2017.8215984

[12] Prashanth Jakkula. [n.d.]. Container Runtime Performance Evaluation of Kubernetes and OpenShift. ([n.d.]).

[13] Zhanibek Kozhirbayev and Richard O Sinnott. 2017. A performance comparison of container-based technologies for the cloud. Future Generation Computer Systems 68 (2017), 175-182.

[14] Endah Kristiani, Chao-Tung Yang, Yuan Ting Wang, and Chin-Yin Huang. 2018. Implementation of an edge computing architecture using openstack and kubernetes. In International Conference on Information Science and Applications. Springer, 675-685.

[15] Nikhil Marathe, Ankita Gandhi, and Jaimeel M Shah. 2019. Docker swarm and kubernetes in cloud computing environment. In $20193 \mathrm{rd} \mathrm{In-}$ ternational Conference on Trends in Electronics and Informatics (ICOEI). IEEE, 179-184.

[16] Víctor Medel, Rafael Tolosana-Calasanz, José Ángel Bañares, Unai Arronategui, and Omer F Rana. 2018. Characterising resource management performance in Kubernetes. Computers \& Electrical Engineering 68 (2018), 286-297.

[17] Roberto Morabito, Jimmy Kjällman, and Miika Komu. 2015. Hypervisors vs. lightweight virtualization: a performance comparison. In 2015 IEEE International Conference on Cloud Engineering. IEEE, 386-393.
[18] Stefano Picozzi, Mike Hepburn, and Noel O'Connor. 2017. DevOps with Openshift: Cloud deployments made easy. " O’Reilly Media, Inc.".

[19] Moritz Raho, Alexander Spyridakis, Michele Paolino, and Daniel Raho. 2015. KVM, Xen and Docker: A performance analysis for ARM based NFV and cloud computing. In 2015 IEEE 3rd Workshop on Advances in Information, Electronic and Electrical Engineering (AIEEE). IEEE, 1-8.

[20] Tasneem Salah, M Jamal Zemerly, Chan Yeob Yeun, Mahmoud AlQutayri, and Yousof Al-Hammadi. 2017. Performance comparison between container-based and VM-based services. In 2017 20th Conference on Innovations in Clouds, Internet and Networks (ICIN). IEEE, $185-190$.

[21] Kyoung-Taek Seo, Hyun-Seo Hwang, Il-Young Moon, Oh-Young Kwon, and Byeong-Jun Kim. 2014. Performance comparison analysis of linux container and virtual machine for building cloud. Advanced Science and Technology Letters 66, 105-111 (2014), 2.

[22] Prateek Sharma, Lucas Chaufournier, Prashant Shenoy, and YC Tay. 2016. Containers and virtual machines at scale: A comparative study. In Proceedings of the 17th International Middleware Conference. 1-13.

[23] Cristian Constantin Spoiala, Alin Calinciuc, Corneliu Octavian Turcu, and Constantin Filote. 2016. Performance comparison of a WebRTC server on Docker versus virtual machine. In 2016 International Conference on Development and Application Systems (DAS). 295-298. https://doi.org/10.1109/DAAS.2016.7492590

[24] Salman Taherizadeh and Marko Grobelnik. 2020. Key influencing factors of the Kubernetes auto-scaler for computing-intensive microservice-native cloud-based applications. Advances in Engineering Software 140 (2020), 102734.

[25] John Paul Walters, Andrew J Younge, Dong In Kang, Ke Thia Yao, Mikyung Kang, Stephen P Crago, and Geoffrey C Fox. 2014. GPU passthrough performance: A comparison of KVM, Xen, VMWare ESXi, and LXC for CUDA and OpenCL applications. In 2014 IEEE 7th international conference on cloud computing. IEEE, 636-643.

[26] Jie Zhang, Xiaoyi Lu, and Dhabaleswar K Panda. 2016. Performance characterization of hypervisor-and container-based virtualization for HPC on SR-IOV enabled InfiniBand clusters. In 2016 IEEE International Parallel and Distributed Processing Symposium Workshops (IPDPSW). IEEE, 1777-1784. 